\title{
CWDM metropolitan multiple-access ring network based on optical packet switching
}

\author{
Alicia López • María Ángeles Losada • \\ Ignacio Garcés · Ignacio de Miguel
}

(C) Springer Science + Business Media, Inc. 2006

\begin{abstract}
In the online version of the original article, there was a typographical error in the title. The correct title is listed above. This error occurred in both the html and pdf versions

of the online article. This was a typesetter's error.
\end{abstract} The online version of the original article can be found at http://dx.doi.org/10.1007/s11107-006-5328-9. 